\title{
LAS ESTRATEGIAS DE APRENDIZAJE Y SUS PARTICULARIADES EN LENGUAS EXTRANJERAS
}

\author{
Mayra Rodríguez Ruiz y Emilio García-Merás García \\ Universidad Central "Marta Abreu" de Las Villas, Cuba
}

\section{ACERCA DEL CONCEPTO DE APRENDIZAJE}

En relación con el aprendizaje se han definido diferentes tendencias que se pueden ubicar entre dos polos: el conductismo y el cognoscitivismo. El primero hace énfasis en las condiciones externas que favorecen el aprendizaje; aquí lo esencial es la respuesta y el reforzamiento de aquellas que se encaminen a lograr el resultado esperado. Por su parte, en la tendencia cognoscitivista lo fundamental es el estudiante con su campo vital, su estructura cognoscitiva y sus expectativas.

En el conductismo el aprendizaje es visto como un proceso gradual de reforzamiento de respuestas, o sea, el objetivo es consolidar esta acción para incorporarla a patrones de conducta existentes. Aquí se hiperboliza el papel del medio pues este representa un conjunto de estímulos y se obvian el papel activo del sujeto y los factores psicológicos que intervienen en este proceso.

Para el cognoscitivismo el aprendizaje es ir más allá de la información obtenida, luego parte de un saber influido y conformado por el hombre. Aquí se destaca el papel activo del sujeto, quien elabora, crea o desarrolla constructos, estructuras, metas o conceptos del mundo que discurre a su alrededor. El sujeto, por tanto, resulta sobredimensionado y se considera que el sujeto todo lo hace sin una influencia o estímulo externo para aprender.

Los partidarios de la Gestalt, escuela muy conocida por sus aportes en los estudios de la percepción, sostenían que el aprendizaje estaba en estrecha relación con la percepción; de ahí que consideraran el aprendizaje como la reorganización del mundo conceptual.

Otra corriente, el humanismo, le atribuye al sujeto que aprende toda la responsabilidad del proceso, en tanto que el docente debe propiciar un ambiente de confianza para garantizar el cambio. Luego entonces en el enfoque humanista se propicia un aprendizaje sin coacción, prescripción o imposición. Tampoco hay un aprendizaje conjunto. Todo el proceso de enseñanza-aprendizaje está regido por las motivaciones de condiciones, de modo que al docente le queda la tarea de formar una conciencia desarrolladora ce la personalidad de cada estudiante.

De acuerdo con el enfoque histórico-cultural de L. S Vigotsky, el aprendizaje es aquel proceso en el cual el individuo asimila determinada experiencia histórico-cultural al mismo tiempo que se apropia de ella. Esto, por supuesto, necesita de un sujeto activo, que le dé sentido a esta experiencia, transformándola en su subjetividad; o sea que el aprendizaje es "un proceso que partiendo de lo externo, del medio como fuente proveedora, se realiza por y en el individuo atendiendo a sus necesidades y a través de la actividad y la comunicación propias y de los otros como portadores estos últimos, de toda la riqueza individual y social." (Febles 1999: 217) 
Según Vigotsky, el aprendizaje en el contexto escolar implica siempre adquisición de conocimiento y construcción de significado. De acuerdo con la tesis vigotskiana del aprendizaje el actor principal del proceso es el estudiante, aunque no el único. El aprendizaje tiene lugar en un sistema interpersonal y, por tanto a través de las interacciones con el docente y con los compañeros del aula, el estudiante aprende los instrumentos cognitivos y comunicativos de su cultura. El objetivo de la teoría de Vigotsky es descubrir y estimular la zona de desarrollo potencial o zona de desarrollo próximo en cada estudiante; en esta teoría se destaca la idea de que el sujeto no se limita a responder a los estímulos de modo pasivo o mecánico sino que actúa sobre ellos.

Uno de los factores que ha hecho que los docente de lenguas extranjeras tiendan a basar su enseñanza en el enfoque socio-cultural es el énfasis que este pone en la mediación en el proceso de aprendizaje. De acuerdo con Vigotsky, es a través de la mediación social que el conocimiento se hace viable y gana coherencia. La mediación constituye entonces un mecanismo mediante el cual las actividades socio-culturales externas se transforman en funcionamiento mental interno.

Cuando se aprende una lengua extranjera esta mediación puede ser un libro, un material visual, el discurso del aula, la instrucción o cualquier otra forma de ayuda del docente. La mediación social en la forma de interacción puede ocurrir entre una persona experta y otra con menos experiencia; en el aula de lengua extranjera esta mediación puede darse entre el docente como experto y el estudiante o entre los propios estudiantes.

Desde la concepción vigostkiana de mediación, el pensamiento humano surge en el contexto de actividades que están presentes en escenarios sociales y culturales específicos. Aplicando este idea al aula, la mediación incluye los medios de enseñanza-aprendizaje; la misma puede ocurrir de manera escrita o hablada, pues ambas formas del lenguaje son importantes para la construcción del conocimiento y el desarrollo cognitivo. El papel mediacional del docente no implica un trabajo o esfuerzo adicional de su parte, sino que su papel adquiere una forma cualitativamente superior y diferente.

El papel del docente desde una visión socio-cultural va más allá de simplemente proveer al estudiante de una nueva lengua, sino que la misma es empleada como una herramienta cognitiva que le permite desarrollar pensamientos e ideas en esa lengua. El proceso de pensamiento es un indicador de desarrollo en los estudiantes quienes son capaces de completar tareas de manera independiente a medida que alcanzan su nivel potencial de desarrollo.

La enseñanza de una lengua extranjera desde una visión socio-cultural no implica sencillamente prestar atención al material lingüístico que se enseña sino además prestar atención al sujeto que aprende, pues los docente deben propiciar a los estudiantes un ambiente de guía y de apoyo además del conocimiento que está por encima del nivel actual que ellos poseen. El significado se construye mediante la actividad conjunta y no mediante la transmisión de conocimiento del docente a los estudiantes; el conocimiento no se transfiere del docente al estudiante sino que se ayuda a este a transformar el conocimiento que recibe mediante la construcción de significado y con recursos como son el conocimiento de sus interlocutores, de los libros de texto y de otros medios en el contexto del aula. 


\section{VALORACIONES TEÓRICAS ACERCA DE LAS ESTRATEGIAS DE APRENDIZAJE}

En años recientes ha existido la tendencia hacia un enfoque estratégico del proceso de enseñanzaaprendizaje en general y de las lenguas extranjeras en particular.

Las estrategias de aprendizaje, según Weinstein y Mayer (1986 p. 315), pueden definirse como "conductas y pensamientos que un aprendiz utiliza durante el aprendizaje con la intención de influir en su proceso de codificación". Para Danserau (1985) y Nisbet y Schucksmith (1987), las estrategias constituyen secuencias integradas de procedimientos o actividades que se eligen con el propósito de facilitar la adquisición, almacenamiento y/o tuilización de la información. Por su parte, otros autores como Beltrán, García-Alcaniz, Moraleda, Calleja y Santuiste (1987) las definen como actividades u operaciones mentales que se emplean para facilitar el conocimiento. Ellos les añaden dos características: que sean directa o indirectamente manipulables y que tengan un carácter intencional o propositito.

Otro notable autor, Monereo (1994), y que nos parece concilia de manera muy acertada estas ideas anteriores, al referirse a las estrategias las define como procesos de toma de decisiones (conscientes e intencionales) en las cuales el estudiante elige y recupera, de manera coordinada, los conocimientos que necesita para cumplimentar una determinada demanda u objetivo, en dependencia de las características de la situación educativa en que se produce la acción.

Tomando como base estas ideas referidas más arriba, podemos afirmar que los autores más representativos en este campo coinciden en resaltar algunos elementos básicos del concepto de estrategias de aprendizaje: que implican una secuencia de actividades, operaciones o planes dirigidos a la consecución de metas de aprendizaje y que tienen un carácter consciente e intencional en el que se implican procesos de toma de decisiones por el estudiante ajustadas al objetivo que pretende alcanzar. Para Beltrán (1993) las definiciones expuestas resaltan dos aspectos importantes al establecer el concepto de estrategias: actividades $u$ operaciones mentales que realiza el estudiante para mejorar el aprendizaje y el carácter intencional que implican un plan de acción.

A modo de resumen, puede verse que los rasgos esenciales que aparecen en la mayor parte de las definiciones sobre estrategias es que son acciones que parten de la iniciativa del estudiante, que están constituidas por una secuencia de actividades, que son controladas por el sujeto que aprende y que son, generalmente, deliberadas y planificadas por el propio estudiante.

\section{LAS ESTRATEGIAS DE APRENDIZAJE DE UNA LENGUA EXTRANJERA}

Las investigaciones relacionadas con las estrategias de aprendizaje de una lengua extranjera datan de los años sesenta del pasado siglo como resultado de los avances alcanzados por la psicología cognitiva, siendo el interés principal identificar lo que los buenos aprendices de una lengua extranjera reportaban que hacían para aprenderla y, en algunos casos, se recurría a su observación durante el proceso de aprendizaje.

En 1966 se publica The Method of Inference in Foreign Language Study de Aaron Carter, obra que constituyó el primer intento relacionado con el tema de estrategias de aprendizaje de una lengua extranjera (Hismanoglou 2000: 1): Posteriormente entre los años setenta, ochenta y noventa se realizaron 
estudios por otros especialistas ente los que sobresalen Tarone (1983), O'Malley y Chamot (1990) y Rebecca Oxford (1990, 1992, 1993) (Lessard Clouston 1997:2)

Tarone (1983, ctdo en Lessard-Clouston, 1997), define las estrategias de aprendizaje de una lengua extranjera como "un intento por desarrollar la competencia lingüística y sociolingüística en la lengua de llegada para incorporar estas dentro de su competencia interlingual." Para Rubin (1987, ctdo en Lessard-Clouston 1997), las estrategias de aprendizaje son "estrategias que contribuyen al desarrollo del sistema de la lengua que el aprendiz construye y afectan directamente el aprendizaje". O'Malley y Chamot (1990: 1) definen las estrategias como "pensamientos o comportamientos especiales que los individuos usan para ayudarse a apropiarse, aprender o retener nueva información."

Finalmente presentamos una definición aportada por Rebecca Oxford y que en nuestra opinión resulta la más completa y útil para el trabajo con una lengua extranjera. Según esta autora las estrategias de aprendizaje de una lengua son "...acciones específicas, comportamientos, pasos o técnicas que los estudiantes (con frecuencia de manera intencional) utilizan para mejorar su progreso en el desarrollo de sus habilidades en la lengua extranjera." $\mathrm{Y}$ sigue planteando que: "Estas estrategias pueden facilitar $\mathrm{a}$ internalización, el almacenamiento, la recuperación o el uso de la nueva lengua. Las estrategias son herramientas necesarias para el desarrollo de habilidades comunicativas." (Oxford 1990, p. 18)

Como puede observarse se ha experimentado un cambio con relación al concepto de estrategias de aprendizaje de lenguas extranjeras, desde aquellas primeras con énfasis en el producto de las estrategias de aprendizaje de lenguas (competencia lingüística o sociolingüística) hacia un énfasis en los procesos y las características de dichas estrategias. Independientemente de que no exista una uniformidad en cuanto a la definición del término estrategias de aprendizaje en lenguas extranjeras, sí existen al menos algunas características comunes (Lessard-Clouston, 1997):

- $\quad$ son generadas por el estudiante

- $\quad$ son pasos que siguen los estudiantes en el proceso de aprendizaje

- mejoran el aprendizaje de la lengua y ayudan a desarrollar la competencia lingüística

- $\quad$ pueden ser visibles (comportamientos, pasos que siguen, técnicas que emplean) o no visibles (pensamientos, procesos mentales)

- incluyen la información y la memoria (conocimiento de vocabulario, reglas gramaticales)

- $\quad$ permiten al estudiante ser más autónomo

- amplían el papel de los docente de lengua

- se orientan hacia un problema

- incluyen muchos aspectos, no solamente el cognitivo

- pueden enseñarse

- $\quad$ son flexibles

- reciben la influencia de una variedad de factores 


\section{CLASIFICACIÓN DE LAS ESTRATEGIAS}

Otro asunto relacionado con las estrategias de aprendizaje se refiere a su clasificación. Así nos vamos a encontrar con un determinado número de clasificaciones partiendo del propio análisis conceptual del término; no obstante aún reconociendo la gran diversidad existente a la hora de categorizar las mismas, suelen existir ciertas coincidencias entre algunos autores al establecer tres grandes clases de estrategias: cognitivas, metacognitivasy de manejo de recursos (Valle Arias et al 1999:442):

Las estrategias cognitivas se refieren a la integración del nuevo material con el conocimiento previo; las metacognitivas hacen referencia a la planificación, control y evaluación por parte de los estudiantes de su propia cognición. Por su parte las estrategias de manejo de recursos son un conjunto de estrategias de apoyo que incluyen diferentes tipos de recursos que contribuyen a que la resolución de la tarea se lleve a buen término. Gran parte de las estrategias incluidas en este tercer grupo tiene que ver con la disposición afectiva y motivacional del sujeto hacia el aprendizaje.

De igual forma en el aprendizaje de una lengua extranjera nos encontramos con un determinado número de clasificaciones o taxonomías de las estrategias de aprendizaje. Así, por ejemplo, Rubin (1987, ctdo en Hismanoglu 2000) considera que existen tres tipos de estrategia que bs aprendices usan y que contribuyen directa o indirectamente al aprendizaje de la lengua: de aprendizaje, de comunicación y sociales. Las primeras contribuyen de forma directa al desarrollo del sistema de la lengua construido por el estudiante, las segundas tienen menor relación con el aprendizaje de la lengua al centrarse en el proceso propio de la comunicación y las terceras contribuyen de manera indirecta pues no conducen directamente a la obtención, almacenamiento, recuperación y uso de la lengua, sino que se relacionan con aquellas actividades en las que el estudiante tiene la oportunidad de exponerse a determinadas situaciones donde verifica lo que ha aprendido.

O'Malley (1990: 44-46) clasifica las estrategias en tres subcategorías principales: las metacognitivas, las cognitivas y las socioafectivas Las estrategias metacognitivas requieren de planificación, reflexión, monitoreo y evaluación del aprendizaje (atención dirigida, atención selectiva, autocontrol y autoevaluación): Las cognitivas están más limitadas a tareas específicas de aprendizaje e implican una manipulación más directa del material de aprendizaje como por ejemplo, la repetición, la traducción, la agrupación de elementos, la toma de notas. Las estrategias socio-afectivas se relacionan con las actividades de mediación y transacción social con otras personas como son el trabajo cooperado y las preguntas aclaratorias.

Según Stern (1992 ctdo en Hismanoglu 2000), existen cinco estrategias para el aprendizaje de una lengua: de planificación y control, cognitivas, comunicativo-experienciales, interpersonales y afectivas.

Para Stern las estrategias de control y planificación se relacionan con la intención del estudiante para dirigir su propio aprendizaje; las estrategias cognitivas son pasos u operaciones usadas en el aprendizaje o la solución de un problema que requiere del análisis directo, la transformación o la síntesis de los materiales de aprendizaje. Con el objetivo de evitar la interrupción en el flujo de la comunicación se usan estrategias comunicativo-experienciales que incluyen los gestos, el parafraseo, entre otras. Las estrategias interpersonales son para Stern las que deben monitorear el propio desarrollo del estudiante y evaluar su propio desempeño y en tal sentido los estudiantes deben contactar con hablantes nativos y 
familiarizarse con la cultura de la lengua objeto de estudio. Por otra parte las estrategias afectivas son aquellas que se relacionan con la actitud que muestran los aprendices hacia la lengua extranjera, sus hablantes y su cultura.

La taxonomía que propone Rebecca Oxford (1990: 16) es, a nuestro juicio, una de las más completas y útiles para trabajar debido a la manera en que las organiza atendiendo a que precisamente las estrategias de aprendizaje de una lengua extranjera tienen como propósito principal el desarrollo de la competencia comunicativa; que constituye a su vez el fundamento del enfoque comunicativo en la enseñanza de las lenguas extranjeras donde el estudiante pasa a desempeñar un papel más activo y de más responsabilidad en el aprendizaje de la lengua extranjera; de ahí que requiera de herramientas específicas entre las que se encuentran las estrategias de aprendizaje.

Esta autora primeramente hace una distinción entre estrategias directas (de memoria, cognitivas y compensatorias) e indirectas (metacognitivas, afectivas y sociales): Las primeras incluyen de forma directa a la lengua extranjera, requieren de su procesamiento mental y se emplean para desarrollar las cuatro habilidades. Las estrategias indirectas, aunque no involucran directamente a la lengua que se estudia, sí son útiles por cuanto sirven para apoyar y controlar el aprendizaje de la lengua en cuestión. Como debe suponerse, los mejores resultados se obtienen cuando existe una adecuada combinación de ambas.

En esta taxonomía las estrategias de memoria son aquellas usadas para almacenar o guardar la información y recuperarla cuando se necesite, las estrategias cognitivas son las estrategias mentales que utilizan los estudiantes para que su aprendizaje sea significativo, o sea, se usan en la formación y revisión de modelos mentales internos y para producir y recibir mensajes en la lengua extranjera; y las estrategias compensatorias ayudan a los estudiantes a eliminar los vacíos de conocimiento y poder dar continuidad a la comunicación.

Por su parte, las estrategias metacognitivas ayudan a los estudiantes a dirigir, planificar, regular y autoevaluar su aprendizaje; las estrategias afectivas se relacionan con la parte emotiva del estudiante pues les permiten controlar sus sentimientos, motivaciones y actitudes relacionadas con el aprendizaje de la lengua, en tanto las estrategias sociales llevan a la interacción creciente con la lengua extranjera al facilitarles la interacción con otros estudiantes, en una situación discursiva.

\section{ENSEÑANZA DE LAS ESTRATEGIAS}

La enseñanza de las estrategias es un asunto polémico por el número de interrogantes y la falta de acuerdo entre diferentes autores respecto al tema. A los problemas asociados a cualquier tipo de enseñanza (qué, cuándo, cómo enseñar) se le añaden otros como, por ejemplo, si las estrategias deben enseñarse separadamente del currículo o junto a él, si deben enseñarse estrategias generales o específicas; así como otros problemas relacionados con los conocimientos y habilidades que debe tener el docente para ser considerado un instructor eficaz.

Somos de la opinión de que las estrategias pueden enseñarse de una forma integrada en las diferentes áreas curriculares y también mediante el uso de otras vías complementarias que pueden ayudar a un mejoramiento en los procesos de pensamiento de los estudiantes y a un mayor conocimiento y control sobre sus recursos, posibilidades y limitaciones cognitivas. 
El segundo problema referente a si se deben enseñar estrategias generales o específicas, consideramos que la enseñanza de las primeras está mucho más relacionada con programas de entrenamiento cognitivo, de enseñar a pensar; por su parte, las estrategias específicas, al estar más relacionadas con las diferentes áreas curriculares, tiene más sentido que se incorporen al currículo escolar y tratando de integrarlas a los objetivos específicos de cada disciplina o área académica.

La otra cuestión señalada como un problema se relaciona con el papel que le corresponde desarrollar al docente. Nos preguntaríamos entonces: ¿Qué es un docente estratégico? Para Beltrán (1993), "docente estratégico" es el término que sirve para definir los rasgos más sobresalientes de un instructor eficaz y que se pueden resumir en las ideas siguientes: un verdadero pensador y un especialista en la toma de decisiones; un experto que posee una amplia base de conocimientos, un verdadero mediador y un modelo para el estudiante. Por tanto, el docente sería estratégico en su aprendizaje y en su acción docente dirigida a influir en los procesos de aprendizaje de los estudiantes.

Analicemos a continuación el otro elemento del proceso de enseñanza- aprendizaje: el estudiante. Habíamos apuntado anteriormente que las estrategias de aprendizaje de una lengua extranjera son acciones específicas, comportamientos o pasos que los estudiantes emplean de manera consciente o no para mejorar su desempeño en la lengua extranjera. Así, por ejemplo, en el aula observamos que los estudiantes utilizan diferentes estrategias para dar cumplimiento a una tarea como consultar al compañero de aula, agrupar las palabras de acuerdo con su empleo, usar gestos, entre otras, Por supuesto todo esto ocurre de manera diferente en cada estudiante pues cada uno tiene su propio estilo de aprendizaje y en consecuencia utiliza unas estrategias $u$ otras.

Teniendo en cuenta algunas de las ideas planteadas con relación a las estrategias de enseñanzaaprendizaje, expondremos a continuación algunas de las principales características de una instrucción estratégica eficaz. De acuerdo con Paris (1988 pp313-316), una instrucción estratégica debe cumplir con lo siguientes requisitos:

- Las estrategias deben ser funcionales y significativas

- La instrucción debe demostrar qué estrategias pueden ser utilizadas, cómo pueden aplicarse y cuándo y por qué son útiles

- Los estudiantes deben creer que las estrategias son útiles y necesarias

- Debe haber una conexión entre la estrategia enseñada y las percepciones del estudiante sobre el contexto de la tarea

- Una instrucción eficaz y con éxito genera confianza y creencias de autoeficiencia

- La instrucción debe ser directa, informativa y explicativa

- La responsabilidad para generar, aplicar y controlar estrategias eficaces es transferida del instructor al estudiante

- Los materiales de instrucción deben ser claros, bien elaborados y agradables

Resumiendo, la enseñanza de estrategias presupone o demanda enseñar a los estudiantes a ser estratégicos, o sea, a ser capaces de actuar de manera intencionada para alcanzar determinados objetivos de aprendizaje teniendo en cuenta las características de la tarea, las exigencias del entorno y las propias 
limitaciones y recursos personales. Por tanto, enseñar a pensar conlleva enseñar al estudiante a emplear de manera estratégica sus capacidades cognitivas, sus técnicas y procedimientos de estudio, adaptándolos a la situación de aprendizaje concreta.

\section{BIBLIOGRAFÍA}

ANTICH DE LEON, R. et al. (1986): Metodología de la enseñanza de lenguas extranjeras. La Habana: Editorial Pueblo y Educación

BEAUQUIS, C. (2000): "Language Learning Strategies: A Pandora's "Black Box"? Should the use of language learning strategies be taught in language classes?" En The Western Journal of Graduate Research, Vol. 9 (1), pp. 51-62

BECCO, G. R. "Vygotsky y teorías sobre el aprendizaje. Conceptos centrales perspectiva vygotskyana". En www.jalisco.gob.mx/srias/educacion/9riolugo.html (Consultada en enero de 2003)

BELTRÁN J. (1993): Procesos, estrategias y técnicas de aprendizaje. Madrid: Síntesis.

Bernal Diaz, P. y Y. Pedraza LinARES. (2003): "Las estrategias de aprendizaje de idioma inglés en estudiantes de medicina. Una fundamentación necesaria". En Memorias II Conferencia Internacional Problemas Pedagógicos de la Educación Superior. Universidad Central "Marta Abreu" de Las Villas.

CAMPS, A. Y M. CASTELló BADíA. (1996): “Las estrategias de enseñanza-aprendizaje en la escritura”. En EI asesoramiento psicopedagógico: una perspectiva profesional y constructivista. Alianza Editorial, S. A., Madrid. Pp.322-333

СНАМОТ, A.U. (1999): Learning Strategy Instruction in the English Classroom. En TLT Online Editor. http://hyper.chubu.ac.jp/jalt/pub/tt/99/jun/chamot.html (Consultada en octubre de 2001)

"Didáctica de las lenguas extranjeras o segundas lenguas". (1998): En Enciclopedia General de la Educación, Tomo 3. España: Océano Grupo Editorial, S. A. pp. 1216-1260

DORADO PEREA, C. "Aprender a Aprender. Estrategias y Técnicas". En www.xtec.es/ cdorado/cdora1/esp. (Consultada en octubre de 2001)

Febles ElEJALDE, M. M. (1999): “Un punto de vista sobre el carácter activo del sujeto del aprendizaje”. En Revista Cubana de Psicología, Vol. 16, No. 3, pp 214-221

GÓMEZ, I. (1999): “Enseñanza y aprendizaje”. En Cuadernos de Pedagogía, No. 250, España, pp 54-59 Hismanoglu, M. (2000): "Language Learning Strategies in Foreign Language Learning and Teaching". En The Internet TESL Journal. Vol. V, No. 8 http://aitech.ac.jp./觹esli/Articles/HismanogluStrategies.html (Consultada en octubre de 2001)

KLINGBERG, L. (1978): Introducción a la Didáctica General. Ciudad de La Habana: Editorial Pueblo y Educación

Lessard-Clouston, M. (1997): 'Language Learning Strategies: An Overview for L2 Teachers". En The Internet TESL Journal. Vol III, No. 12, December, http://aitech.ac.jp.//iteslj/Articles/LessardClouston.html (Consultada en octubre de 2001) 
LITTLEWOOD, W. (1986): Communicative Language Teaching: An Introduction. London: C.U.P.

MARTín-Molero, F. (1998): Capítulo 2. "Conceptualización de Estrategias de Enseñanza-Aprendizaje". En Estrategias de Enseñanza y Aprendizaje. (Coordinadora María Luisa Sevillano García) Universidad Nacional de Educación a Distancia. Madrid: España (pp. 73-119)

MONEREO, C. (1994): Estrategias de enseñanza y aprendizaje. Formación del profesorado y aplicación en el aula. España: Editorial Graó

O'MALLEY, J. M. Y ANN UHL CHAMOT. (1990): Learning Strategies in Second Language Acquisition. United States of America: C.U.P.

OXFORD, R. (2000): "Good Language Learners". En Language Learning Strategies: An Update. ERIC Digest.

(1990): Language Learning Strategies: What Every Teacher Should Know. USA: Heinle \& Heinle Publishers. pp. 16-21; 317-330

Rodríguez AROCHO, W. C. (1999): "El legado de Vygotsky y de Piaget a la educación". En Revista Latinoamericana de Psicología, Vol. 31, No. 3, pp. 477-489

SCARCELLA, R. C. Y REBECCA L. OXFORD. (1992): The Tapestry of Language Learning: The Individual in the Communicative Classroom. Boston: Heinle \& Heinle Publishers. pp. 110-120

SILVESTRE ORAMAS, M. (1999): Aprendizaje, educación y desarrollo. La Habana: Editorial Pueblo y Educación

SILVESTRE ORAMAS, M Y PILAR RICO. (1996): Proceso de enseñanza-aprendizaje. Breve referencia del estado actual del problema. Ciudad de La Habana: MINED

TURNER MARTí, L. (1989): Se aprende a aprender. La Habana: Editorial Pueblo y Educación

VALERA MÉNDEZ, R. (1998): Capítulo 8. "Estrategias de Enseñanza-Aprendizaje de idiomas Extranjeros". En Estrategias de Enseñanza y Aprendizaje. (Coordinadora María Luisa Sevillano García) Universidad Nacional de Educación a Distancia. Madrid: España (pp. 345-411)

Valle ArIAS, A., et al. "Las estrategias de aprendizaje. Revisión teórica y conceptual". En Revista Latinoamericana de Psicología, Vol. 31, No. 3, pp. 425-461

VIGOTSKY, L. S. (1964): Lenguaje y Pensamiento. Buenos Aires: Ed. Lautaro 


\title{
Contactar
}

Revista lberoamericana de Educación

\author{
Principal OEI
}

